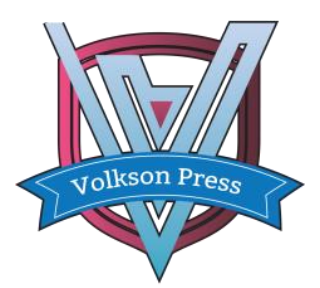

Contents List available at VOLKSON PRESS

Engineering \& Technology Innovations (ETI)

DOI : http://doi.org/10.26480/iceti.01.2017.11.15

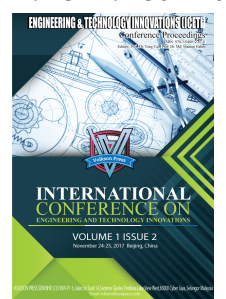

\title{
INTERACTIVE STUDY ON THE RELATIONSHIP BETWEEN RESIDENCE AND CITY TRAFFIC SYSTEM BASED ON FUZZY LOGIC
}

Lin Zhang. Xiao Yan. *, Fan Li. Xiaoqing Huang. ${ }^{+}$

*College of Mathematics and Information Science, Nanchang Hangkong University, 330063, Nanchang, Jiangxi, China

This is an open access article distributed under the Creative Commons Attribution License, which permits unrestricted use, distribution, and reproduction in any medium, provided the original work is properly cited

\section{ARTICLE DETAILS}

\section{Article History:}

Received 02 october 2017 Accepted 06 october 2017 Available online 11 october 2017

\section{Keywords:}

Transportation; Fuzzy membership degree; Satisfaction evaluation Residence; Supporting facilities.

\section{ABSTRACT}

In order to study the influence of the choice of residence to urban traffic, a comprehensive evaluation model of urban residents' to choosing their residence is established. Due to the difficulty in quantifying many facilities around the residence, this paper obtained the fuzzification degree of the facilities' completeness around the the residential area by way of fuzzification means of the facilities' completeness. The fuzzy comprehensive evaluation model of each district in the city is put forward to ccombine with the residential price and the average income of urban residents. What's more, we simulated a medium-sized city, and verify the parameters of the model through MATLAB. Examples show that: A city which is of little poor facilities' completeness can lead some traffic jam to that of comparatively better facilities' completeness in certain degree. It is obvious that the choice of residence plays a significant role in urban traffic.

\section{INTRODUCTION}

The With the rapid development of Chinese economy and the increase of the urban population, a large amount of rural people swarm into cities and the city residential population is expanding gradually. All of the above lead to the sharp expansion of residence and a series of traffic problem. The residential selection and the city traffic system is a kind of nonmalignant interactive system. Once the residence is decided, different transportation will be established and it will affect the residents' selection to their residence in turn. Good choice of the residence can be convenient to the transportation of family shopping, entertainment and commuting, etc. From the perspective of the system, the good planning of residence can reduce the usage amount of urban motor vehicles fundamentally, reduce the distance for residents and reduce the requirements for transportation. Therefore, good residence is so important and necessary. Of course, a good traffic system can lead people to choose the residence and optimize the system between residence and city traffic. A city could have a more longterm development if it could deal with the relationship between urban residential land and urban transportation system.

Many domestic experts and scholars have made a deep study on the relationship between residential location and traffic system. Now the research results are mainly divided into two categories [1-7]: the mathematical programming model and the random utility model. The mathematical programming model mainly means that residents choose residence based on the principle of cost minimization maximization. This method is simple and has clear goal, but the city residents will think too much when choosing. Only a mathematical programming model would be difficult to cover all factors comprehensively. Therefore, there will be some certain deviation when using this mathematical programming model to simulate the choice of residential land. The random utility model is an extension of typical linear model. It means: fixed regression coefficient which is regarded as random variables is to describe the relationship between residence and traffic system. This model use a lot of indicators to describe the residence' features and generalize the fixed effect to random effect, considering a large number of individual factors. But the model ignores the interaction of different families, and does not reflect to optimize residents' choosing residential land. Due to many factors they think about, many urban residents could not choose their residence in a whole aspects,even have no idea about residence.

In order to make qualitative factors that are difficult to quantify accurately reflect the important role it plays in choosing residence, this paper, will use fuzzy mathematics to deal with relative qualitative factors in qualified way, and discuss the choice of residence. Through the establishment of mathematical model, we want to conclude the fuzzy comprehensive evaluation to residence to study the influence of selection of residence to city traffic system.

\section{Satisfaction evaluation model of household income and housing} price to residence

Factors such as household income and housing price tend to be the first factor for urban residents to choose residence as a result of different housing prices and urban residents' household income of the city. If the average monthly income of the city family is $P_{j}$, The housing price of the target area $i$ is $N_{i}$ (among $0<i \leq M$, expresses the total number of residence in the city) So the optional residential sets of a family must be (expresses the proportion that is to buy or rent the house in monthly average household income of the city). So we can get the satisfaction evaluation model of housing price to residence:

$$
P_{i}=\frac{\gamma P_{j}-N_{i}}{\gamma P_{j}}
$$

$\left(0 \leq P_{i}<1\right)$

3. The fuzzy membership degree of facilities for completeness around residence

The facilities for completeness around residence plays an important role in the selection of the residential area. Residents will think a lot: school district housing. Hospital, shopping mall, park and other factors. Poor facilities for completeness around residence is not convenient to people's life. In other words, a good residence had better have a relative facility for completeness around residence.

Suppose that a facility for completeness around residence $i$ is 
$u_{i}=\left(u_{i}^{1}, u_{i}^{2}, u_{i}^{3}, u_{i}^{4}\right),\left(u_{i}^{1}, u_{i}^{2}, u_{i}^{3}\right.$ and $u_{i}^{4}$ denote scale evaluation of schools, hospitals, shopping malls). Schools and hospitals were set in a certain level in accordance with the provisions of the state standards. In this paper, the school and the hospital are divided into five grades. According to the district around the school or hospital grade, we define the fuzzy membership degree of facilities for completeness around residence. Its fuzzy language can be expressed as:

[Class $\mathrm{A}]=\frac{1}{1}+\frac{0.9}{0.9}+\frac{0.6}{0.8}+\frac{0.1}{0.7}$

$[$ Class B $]=\frac{0.1}{1}+\frac{0.5}{0.9}+\frac{1}{0.8}+\frac{0.8}{0.7}+\frac{0.1}{0.6}$

$[$ Class $\mathrm{C}]=\frac{0.1}{0.8}+\frac{0.5}{0.7}+\frac{1}{0.6}+\frac{0.8}{0.5}+\frac{0.1}{0.4}$

[Class D] $=\frac{0.1}{0.6}+\frac{0.5}{0.5}+\frac{1}{0.4}+\frac{0.8}{0.3}+\frac{0.1}{0.2}$

[Class $\mathrm{E}]=\frac{0.1}{0.4}+\frac{0.5}{0.3}+\frac{1}{0.2}+\frac{0.8}{0.1}$

Therefore, we can obtain the fuzzy membership degree of facilities for completeness of the school $A\left(u_{i}^{1}\right)$ and the hospital $A\left(u_{i}^{2}\right)$ according to the above formula (2)-(6) . The evaluation of shopping malls and parks is relative to these area. parks and other facilities for the evaluation with the area of these facilities related. Large shopping malls less than $1500 \mathrm{~m}^{2}$ or between 1500 to $7000 \mathrm{~m}^{2}$, or more than $7000 \mathrm{~m}^{2}$ will be divided like the following according to the business area of hypermarket, comprehensive supermarket and convenient store, the fuzzy membership degree of the consumption facilities for completeness can be expressed as:

$$
A\left(u_{i}^{3}\right)=\left\{\begin{array}{cc}
\alpha_{1}\left(\frac{\tilde{u}_{i}^{3}}{1500}\right)^{2} & \tilde{u}_{i}^{3}<1500 \\
1-\left(1-\alpha_{1}\right)\left(\frac{\tilde{u}_{i}^{3}-7000}{5500}\right)^{2} & 1500 \leq \tilde{u}_{i}^{3}<7000 \\
1 & \tilde{u}_{i}^{3} \geq 7000
\end{array}\right.
$$

$\tilde{u}_{i}^{3}$ means the operating area (The units was $m^{2}$ ) of consumption facilities of shopping malls around residence $i$. When $\alpha_{1}=0.8$, the fuzzy membership degree of consumption facilities for completeness of shopping malls around residence $A\left(u_{i}^{3}\right)$ is shown in Fig.1 :

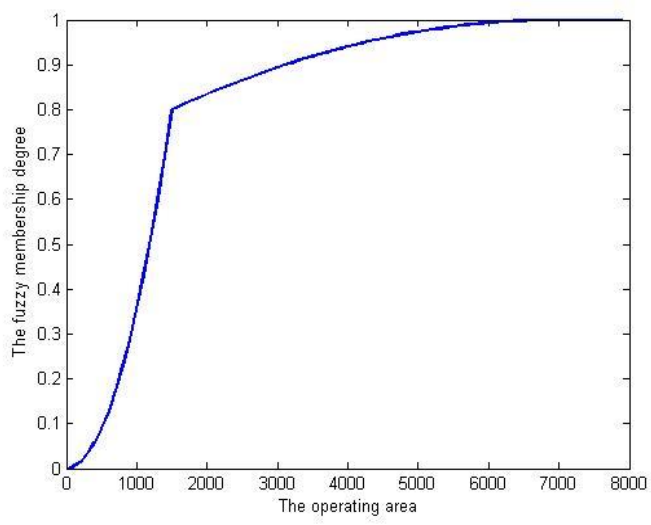

Fig.1 The fuzzy membership degree change of the shopping center From Fig.1 we can see : When $\alpha_{1}=0.8$; the operating area (The units was $\mathrm{m}^{2}$ ) of consumption facilities of shopping malls around residence is less than $1500 \mathrm{~m}^{2}$, the fuzzy membership degree of consumption facilities for completeness of shopping malls around residence increases with the increase of business area; When the operating area is up to $1500 \mathrm{~m}^{2}$, the consumption facilities could meet urban residents in a large degree, so the corresponding fuzzy membership degree is to 0.8 . But when the operating area is between $1500 \mathrm{~m}^{2}$ and $7000 \mathrm{~m}^{2}$, the fuzzy membership degree rise relatively slow; When the operating area is more than $7000 \mathrm{~m}^{2}$, the fuzzy membership degree reaches 1 .

According to the requirements of floor space of large parks, regional parks and small neighborhood parks, we divide the floor space of the researching park in the following way: more than $20 \mathrm{hm}^{2}, 2 \mathrm{hm}^{2} \sim 20$ $\mathrm{hm}^{2}$ and $2 \mathrm{hm}^{2}$ [8].So the fuzzy membership degree of the leisure facilities for completeness of parks around the residence can be expressed as:

$$
A\left(u_{i}^{4}\right)=\left\{\begin{array}{cc}
\alpha_{2}\left(\frac{\tilde{u}_{i}^{4}}{2}\right) & \tilde{u}_{i}^{4} \leq 2 \\
1-\left(1-\alpha_{2}\right)\left(\frac{\tilde{u}_{i}^{4}-20}{18}\right)^{2} & 2<\tilde{u}_{i}^{4} \leq 20 \\
1 & \tilde{u}_{i}^{4} \geq 20
\end{array}\right.
$$

$\tilde{u}_{i}^{4}$ means the area covered (The units was $h m^{2}$ ) of leisure facilities of parks around residence $i$.When $\alpha_{2}=0.4$; the fuzzy membership degree of leisure facilities for completeness of parks around residence $A\left(u_{i}^{4}\right)$ is shown in Fig.2 :

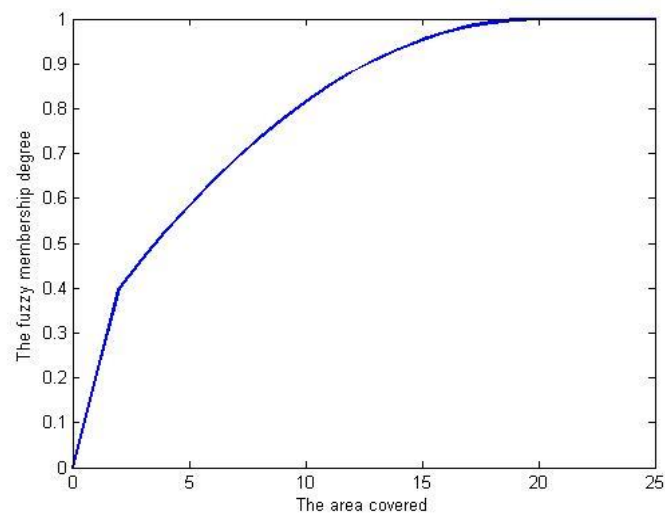

Fig.2 The fuzzy membership degree change of the park

From Fig. 2 we can see : when $\alpha_{2}=0.4$, the area covered（The units was $\mathrm{hm}^{2}$ ) of leisure facilities of parks around residence is less than 2 $\mathrm{hm}^{2}$, the fuzzy membership degree of leisure facilities for completeness of parks around residence increases rapidly with the increase of business area; When the area covered is between $2 \mathrm{hm}^{2}$ and $20 \mathrm{hm}^{2}$, the leisure facilities rise relatively slow, and when the area covered is $5 \mathrm{hm}^{2}$, it could almost meet the needs of most of the city's residents leisure and entertainment needs, the fuzzy membership degree was close to 0.6 ; When the area covered is more than $20 \mathrm{hm}^{2}$, the leisure facilities will be very completed, the fuzzy membership degree reaches 1 .

4 Comprehensive evaluation of the residence surrounding facilities From the above, the matrix of relation $R$ of level of the fuzzy membership of facilities for completeness of $M$ community can be:

$R=\left[\begin{array}{cccc}A\left(u_{1}^{1}\right) & A\left(u_{2}^{1}\right) & \cdots & A\left(u_{M}^{1}\right) \\ A\left(u_{1}^{2}\right) & A\left(u_{2}^{2}\right) & \cdots & A\left(u_{M}^{2}\right) \\ \vdots & \vdots & \vdots & \vdots \\ A\left(u_{1}^{4}\right) & A\left(u_{2}^{4}\right) & \cdots & A\left(u_{M}^{4}\right)\end{array}\right]$ 
Then we should determine whether the area has the above four infrastructure construction and conclude $0-1$ line vector quantity

$W=\left(\begin{array}{llll}w_{1 i} & w_{2 i} & \cdots & w_{\alpha i}\end{array}\right)$

Among

$w_{\alpha i}= \begin{cases}0 & \text { There were corresponding facilities around the district } \\ 1 & \text { There was no corresponding facilities around the district }\end{cases}$

According to $0-1$ line vector quantity $W$ and matrix of relation $R$ of the level of the fuzzy membership degree of facilities for completeness around residence matrix $R$, it can obtain various supporting satisfaction model facilities for completeness of each district :

$$
\begin{aligned}
& A(u)=\frac{1}{4} W \cdot R \\
& =\frac{1}{4}\left(\begin{array}{llll}
w_{1 i} & w_{2 i} & \cdots & w_{\alpha i}
\end{array}\right) \cdot\left[\begin{array}{cccc}
A\left(u_{1}^{1}\right) & A\left(u_{2}^{1}\right) & \cdots & A\left(u_{M}^{1}\right) \\
A\left(u_{1}^{2}\right) & A\left(u_{2}^{2}\right) & \cdots & A\left(u_{M}^{2}\right) \\
\vdots & \vdots & \vdots & \vdots \\
A\left(u_{1}^{4}\right) & A\left(u_{2}^{4}\right) & \cdots & A\left(u_{M}^{4}\right)
\end{array}\right]
\end{aligned}
$$

After calculation, we can get a line of satisfaction value of each district in the city:

$$
A(u)=\left(\begin{array}{llll}
A\left(u_{1}\right) & A\left(u_{2}\right) & \cdots & A\left(u_{M}\right)
\end{array}\right)
$$

In a word, according to the average monthly income of the city family and the evaluation model $P_{i}$ of each residence, combining with the satisfaction model facilities for completeness of each district $A(u)$, we can get the conclusion of ambiguous comprehensive evaluation model of satisfaction degree of each district:

$Z_{i}=\alpha_{3} p_{i}+\alpha_{4} A\left(u_{i}\right)$

$\alpha_{3}, \alpha_{4}$ means the weight of the model $P_{i}$ and model $A(u)$ in the comprehensive evaluation (among $\alpha_{3}+\alpha_{4}=1$ ).

\section{Example}

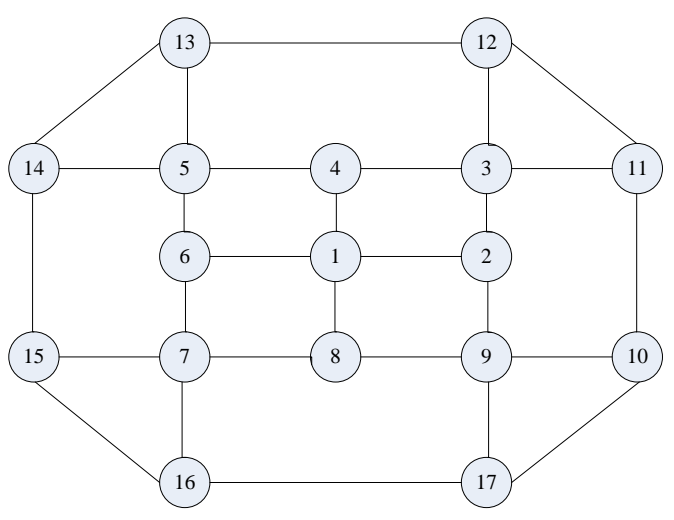

\section{Fig.3 Simple traffic network}

In Fig.3: A medium-sized city is divided into 17 residential areas and Residence 1 is the city center; each residence is connected by a. So we define that the free-flow speed of the two-way multilane which connect each district is equal to road width. The static date of simple traffic network in each residence is in Tab. 1

\begin{tabular}{|c|c|c|c|c|c|c|}
\hline \multicolumn{2}{|c|}{ Length $(\mathrm{km})$} & 2 & 2.5 & 2.5 & 3 & 2.2 \\
\hline \multicolumn{2}{|c|}{ Capacity $\left(v e h \cdot h^{-1}\right)$} & 2000 & 2500 & 2000 & 1000 & 3000 \\
\hline \multicolumn{2}{|c|}{$\begin{array}{l}\text { Free flow velocity ( } \\
\mathrm{km} \cdot \mathrm{h}^{-1} \text { ) }\end{array}$} & 30 & 40 & 30 & 10 & 50 \\
\hline 2,9 & 3,4 & 3,11 & 3,12 & 4,5 & 5,6 & 5,13 \\
\hline 3.5 & 2.8 & 3.5 & 3 & 2 & 1.8 & 3.5 \\
\hline 2000 & 3000 & 4000 & 4000 & 3000 & 2500 & 3500 \\
\hline 30 & 50 & 60 & 60 & 50 & 30 & 60 \\
\hline 5,14 & 6,7 & 7,8 & 7,15 & 7,16 & 8,9 & 9,10 \\
\hline 3 & 2.5 & 2.5 & 3 & 2.8 & 3 & 2.8 \\
\hline 3000 & 2000 & 2000 & 3000 & 4000 & 1500 & 3000 \\
\hline 50 & 30 & 30 & 50 & 60 & 20 & 50 \\
\hline 9,17 & 10,11 & 10,17 & 11,12 & 12,13 & 13,14 & 14,15 \\
\hline 3.2 & 4 & 3 & 3.5 & 5 & 2.9 & 4.5 \\
\hline 4000 & 4000 & 3500 & 4000 & 4000 & 4000 & 4000 \\
\hline 60 & 60 & 60 & 60 & 60 & 60 & 60 \\
\hline 15,16 & 16,17 & & & & & \\
\hline 3.2 & 5 & & & & & \\
\hline 3000 & 4000 & & & & & \\
\hline 50 & 60 & & & & & \\
\hline
\end{tabular}

Tab.1 Static date of simple traffic network

\begin{tabular}{llllll} 
Road & 1,2 & 1,4 & 1,6 & 1,8 & 2,3 \\
\hline
\end{tabular}

Tab.2: The accommodation number of households in each residence, the proportion of high-income families in the area and the number and the

\begin{tabular}{|c|c|c|c|c|c|c|c|}
\hline \multicolumn{3}{|c|}{ Residential location } & 1 & 2 & 3 & 4 & 5 \\
\hline \multicolumn{3}{|c|}{ Family quantity } & 25000 & 16000 & 22000 & 18000 & 23000 \\
\hline \multicolumn{3}{|c|}{$\begin{array}{l}\text { High-income } \\
\text { families/\% }\end{array}$} & $30 \%$ & $25 \%$ & $23 \%$ & $23 \%$ & $20 \%$ \\
\hline \multirow{13}{*}{\multicolumn{2}{|c|}{$\begin{array}{l}\text { Distribution } \\
\text { of the main } \\
\text { destination } \\
\text { of the family }\end{array}$}} & 1 & 5000 & 2000 & 1400 & 1800 & 1400 \\
\hline & & 3 & 2000 & 1800 & 2500 & 1500 & 1200 \\
\hline & & 5 & 2000 & 500 & 1200 & 1500 & 2500 \\
\hline & & 7 & 1000 & 400 & 300 & 400 & 1100 \\
\hline & & 9 & 1000 & 1300 & 1000 & 300 & 300 \\
\hline & & $1 、 3$ & 2000 & 2000 & 1200 & 1400 & 1000 \\
\hline & & 1、 5 & 3000 & 400 & 900 & 1200 & 1500 \\
\hline & & 1、 7 & 1000 & 500 & 300 & 400 & 1000 \\
\hline & & 1、 9 & 1000 & 1200 & 800 & 500 & 300 \\
\hline & & $3 、 5$ & 1000 & 500 & 1200 & 1300 & 1000 \\
\hline & & 3、 9 & 900 & 1300 & 1300 & 500 & 300 \\
\hline & & 5、 7 & 700 & 300 & 200 & 400 & 1400 \\
\hline & & 7、 9 & 700 & 600 & 300 & 200 & 400 \\
\hline 6 & \multicolumn{2}{|l|}{7} & 8 & 9 & 10 & \multicolumn{2}{|c|}{11} \\
\hline 15000 & \multicolumn{2}{|c|}{20000} & 15000 & 18000 & 12000 & \multicolumn{2}{|c|}{10000} \\
\hline $25 \%$ & \multicolumn{2}{|c|}{$22 \%$} & $23 \%$ & $20 \%$ & $18 \%$ & \multicolumn{2}{|c|}{$18 \%$} \\
\hline 2000 & \multicolumn{2}{|c|}{1000} & 1800 & 1200 & 500 & \multicolumn{2}{|c|}{600} \\
\hline 800 & \multicolumn{2}{|c|}{400} & 800 & 1000 & 800 & \multicolumn{2}{|c|}{1600} \\
\hline 1200 & \multicolumn{2}{|c|}{600} & 700 & 600 & 200 & \multicolumn{2}{|c|}{500} \\
\hline 1300 & \multicolumn{2}{|c|}{2000} & 1400 & 1000 & 800 & \multicolumn{2}{|c|}{300} \\
\hline 600 & \multicolumn{2}{|c|}{800} & 1600 & 2000 & 1500 & \multicolumn{2}{|c|}{800} \\
\hline 800 & \multicolumn{2}{|c|}{400} & 400 & 1200 & 600 & \multicolumn{2}{|c|}{1000} \\
\hline 1800 & \multicolumn{2}{|c|}{800} & 400 & 400 & 300 & 80 & \\
\hline 1500 & & & 1600 & 600 & 200 & 30 & \\
\hline 600 & 50 & & 1800 & 1500 & 800 & 80 & \\
\hline 300 & 40 & & 200 & 300 & 200 & 10 & \\
\hline 200 & 20 & & 400 & 1400 & 900 & 80 & \\
\hline 1500 & & & 400 & 200 & 300 & 20 & \\
\hline 600 & & & 1400 & 1600 & 800 & 20 & \\
\hline 12 & 13 & & 14 & 15 & 16 & 17 & \\
\hline 8000 & & & 11000 & 10000 & 8000 & 80 & \\
\hline $12 \%$ & 13 & & $15 \%$ & $17 \%$ & $14 \%$ & 15 & \\
\hline 300 & 30 & & 600 & 600 & 200 & 20 & \\
\hline 2000 & 60 & & 600 & 100 & 100 & 80 & \\
\hline 800 & & & 2000 & 900 & 1000 & 10 & \\
\hline 200 & 30 & & 800 & 1800 & 1400 & 80 & \\
\hline 600 & 10 & & 200 & 600 & 1200 & 18 & \\
\hline 300 & 40 & & 600 & 200 & 100 & 30 & \\
\hline 300 & & & 700 & 600 & 200 & 10 & \\
\hline 100 & 30 & & 800 & 1000 & 300 & 30 & \\
\hline 100 & 20 & & 200 & 600 & 200 & 40 & \\
\hline
\end{tabular}
distribution of destination in each family:

Tab.2.Static data of each residential location 


\begin{tabular}{llllll}
\hline 800 & 800 & 1200 & 200 & 100 & 100 \\
\hline 400 & 200 & 200 & 200 & 100 & 900 \\
\hline 300 & 800 & 600 & 800 & 600 & 100 \\
\hline 100 & 100 & 100 & 500 & 600 & 800
\end{tabular}

\begin{tabular}{lllllll}
0.29 & 0.24 & 0.33 & 0.26 & 0.36 & 0.36 & 0.39 \\
0 & 0.60 & 0 & 0.60 & 0 & 0.20 & 0 \\
0.40 & 0 & 0.40 & 0.60 & 0.40 & 0 & 0.20 \\
0.97 & 0.99 & 0.51 & 0.97 & 0.36 & 0.80 & 0.13 \\
0.78 & 0.64 & 0.78 & 0.53 & 0.81 & 0.64 & 1 \\
0.54 & 0.56 & 0.42 & 0.68 & 0.39 & 0.41 & 0.33 \\
\hline 0.39 & 0.37 & 0.37 & 0.43 & 0.37 & 0.38 & 0.37
\end{tabular}
basic infrastructure such as education facilities such as schools, shopping centers, parks or not its have or not around the residential area (National key schools and third A hospital are Class A according to the state regulations; The provincial key schools and third B Hospital are Class B; The city's key schools and third C hospitals are Class C; The county's key schools and Second grade Hospital are Class D; The general school and the first level hospitals are Class E).

Tab.3 Static data around the facilities in the residential location

\begin{tabular}{|c|c|c|c|c|c|c|c|c|c|}
\hline \multicolumn{5}{|c|}{ Residential location } & 1 & 2 & 3 & 4 & 5 \\
\hline \multicolumn{5}{|c|}{ Price $\left(y u a n \cdot m^{2}\right)$} & 6000 & 5000 & 5500 & 4800 & 5200 \\
\hline \multirow{2}{*}{\multicolumn{2}{|c|}{ School }} & \multicolumn{3}{|c|}{ Have or not } & 1 & 1 & 1 & 0 & 1 \\
\hline & & \multicolumn{3}{|c|}{ Grade } & B & $\mathrm{D}$ & $\mathrm{C}$ & $*$ & $\mathrm{~B}$ \\
\hline \multirow{2}{*}{\multicolumn{2}{|c|}{ Hospital }} & \multirow{2}{*}{\multicolumn{3}{|c|}{$\begin{array}{l}\text { Have or not } \\
\text { Grade }\end{array}$}} & \multirow{2}{*}{$\begin{array}{l}1 \\
\mathrm{~A}\end{array}$} & 0 & 1 & 1 & 0 \\
\hline & & & & & & $*$ & $\mathrm{~B}$ & $\mathrm{C}$ & $*$ \\
\hline \multirow{2}{*}{\multicolumn{2}{|c|}{ Mall }} & \multicolumn{3}{|c|}{ Have or not } & 1 & 1 & 1 & 1 & 1 \\
\hline & & \multicolumn{3}{|c|}{ Selling area } & 8000 & 3000 & 5000 & 2500 & 6000 \\
\hline \multirow[b]{2}{*}{ Park } & \multicolumn{4}{|c|}{ Have or not } & \multirow{2}{*}{$\begin{array}{l}1 \\
1.8\end{array}$} & 1 & 1 & 1 & 1 \\
\hline & \multicolumn{4}{|c|}{$\begin{array}{l}\text { Area } \\
\text { covered }\end{array}$} & & 6 & 5 & 8 & 3 \\
\hline 6 & 7 & & 8 & 9 & & 10 & 11 & 12 & 13 \\
\hline 5000 & 53 & & 4700 & & 200 & 4500 & 4500 & 4300 & 4300 \\
\hline 0 & 1 & & 0 & 1 & & 0 & 1 & 0 & 1 \\
\hline * & $\mathrm{C}$ & & $*$ & C & & $*$ & $\mathrm{E}$ & $*$ & E \\
\hline 1 & 0 & & 1 & 1 & & 1 & 0 & 1 & 0 \\
\hline D & $*$ & & D & C & & D & $*$ & $\mathrm{E}$ & $*$ \\
\hline 1 & 1 & & 1 & 1 & & 1 & 1 & 1 & 1 \\
\hline 5000 & 60 & & 1200 & & 000 & 1000 & 1500 & 600 & 7500 \\
\hline 1 & 1 & & 1 & 1 & & 1 & 1 & 1 & 0 \\
\hline 9 & 6 & & 9 & 4 & & 10 & 6 & 22 & $*$ \\
\hline 14 & & 15 & & 16 & & 17 & & & \\
\hline 4500 & & 450 & & 400 & & 4300 & & & \\
\hline 0 & & 0 & & 0 & & 1 & & & \\
\hline * & & * & & * & & $\mathrm{E}$ & & & \\
\hline 1 & & 1 & & 0 & & 0 & & & \\
\hline $\mathrm{E}$ & & D & & * & & * & & & \\
\hline 1 & & 1 & & 1 & & 1 & & & \\
\hline 800 & & 100 & & 800 & & 1000 & & & \\
\hline 1 & & 1 & & 0 & & 1 & & & \\
\hline 3 & & 5 & & $*$ & & 3 & & & \\
\hline
\end{tabular}

Table. 3 The business area and the area covered of the units were $m^{2}$ and $\mathrm{hm}^{2}$. During the study, the average monthly income of the urban cities was 10000 yuan, and the $\gamma$ was 0.7 . So from the above table, we get the satisfaction of the infrastructure and the fuzzy comprehensive evaluation about the residence in the city's commuting according to housing price and the having or not and the scale of the infrastructure around the residence and use MATLAB2009 simulated model.

\section{Tab.4 Fuzzy evaluation of residence satisfaction}

\begin{tabular}{llllll}
\hline Residential location & 1 & 2 & 3 & 4 & 5 \\
\hline Price satisfaction & 0.14 & 0.29 & 0.21 & 0.31 & 0.26 \\
School satisfaction & 0.80 & 0.40 & 0.60 & 0 & 0.80 \\
Hospital satisfaction & 1 & 0 & 0.80 & 0.60 & 0 \\
Mall satisfaction & 1 & 0.89 & 0.97 & 0.87 & 0.99 \\
Park satisfaction & 0.36 & 0.64 & 0.58 & 0.73 & 0.46 \\
Facilities evaluation & 0.79 & 0.48 & 0.74 & 0.55 & 0.56 \\
Fuzzy synthetic & 0.40 & 0.37 & 0.42 & 0.41 & 0.38 \\
evaluation & & & & &
\end{tabular}

[2] Yulong Pei, Huizhi Xu. Planning method of road network density based on urban area position potential energy [J]. China Journal of Highway and Transport, 2007, 20(3):81-85.

[3] Xiaoyan Li, Hong Chen. Urban eco-transportation planning theory [J].Journal of Chang'an University: Natural Science Edition, 2006 26(1):79-82.

[4] Hualan Wang, Wei Zhou, Yuanqing Wang. Influence of transportation between central city and satellite city on urban spatial expansion [J]. Journal of Chang'an University: Natural

Science Edition, 2007, 27(4):79-83. Although the city center residence 1 has all sorts of facilities, its housing price is higher than other areas. So the fuzzy comprehensive evaluation is residence 8 are similar to residence $2,4,6$, but the poor residentia facilities of residence 8 make its fuzzy comprehensive evaluation slightly lower than the residence $2,4,6$. High-income families living in residence 8 would choose to live in residential area 1,9 which has relatively high fuzzy comple urban traffic congestion. Building a school and expand the supermarket from $1200 \mathrm{~m}^{2}$ to $2000 \mathrm{~m}^{2}$ greatly reduce the ability of residents transportation. Therefore, the managers can adjust to some appropriate measures according to the supporting facilities evaluation of urban optimize the urban space and traffic.

\section{Conclusions}

This paper mainly discusses the interactive relationship between the choice of residence and the transportation system based on the theory of fuzzy mathematics, . By establishing the relationship model between the residents. Then, we gain the fuzzy comprehensive evaluation of each residence combining with the completeness of various facilities around the residence. At the same time, the managers can make reasonable 列 fundamentally and lead the residence and city traffic toward more benign development. Finally, I'd like to say that next step is to explore the influence of open district to the choice of the residence for city residents and city transportation.

\section{Acknowledgements}

This research is supported by National Natural Science Foundation of China under Grant No. 51368046 and Natural Science Foundation of

\section{References}

[1] Huapu Lu, Jianwei Wang, Hong Yuan. Study of optimal urban landuse pattern based on transportation efficiency [J]China Journal of Highway and Transport, 2005, 18(3):109-113. 
[5] Xiaolin Dong, Cheng Song, Jin Zhou, et al. Development of district environment economics indicator system [J]. Journal of Chang'an University: Natural Science Edition, 2008,28(1):87-91.

[6] Wei Zhou, Yuanyuan Li. Developing theory and method of urban public traffic in Chian[J]. Journal of Chang'an University: Natural Science Edition, 2007,27(4):65-69.
[7] Kuanmin Chen, Dake Fan, Chaoqun Ma. Calculation of urban rail transit network scale based on passenger distributing centers[J]. Journal of Chang'an University: Natural Science Edition, 2009, 29(6):78-81.

[8] Park green space management and facilities maintenance manual, Ministry of the Interior Ministry of construction, 1999. 